\title{
Unearthing the electroweak structure of heavy charged gauge bosons
}

\author{
Abhishek M. Iyer ${ }^{1}$ and K. Sridhar ${ }^{2}$ \\ ${ }^{1}$ INFN-Sezione di Napoli, Via Cintia, 80126 Napoli, Italy \\ ${ }^{2}$ Department of Theoretical Physics, Tata Institute of Fundamental Research, \\ Homi Bhabha Road, Colaba, Mumbai 400 005, India
}

(Received 29 November 2018; revised manuscript received 20 April 2019; published 17 July 2019)

\begin{abstract}
Heavy charged bosons, with masses in the range of a few $\mathrm{TeV}$, are a characteristic of several extensions of the Standard Model fields. They could either be embedded in a Standard Model gauge structure or an extended gauge symmetry. Depending on the underlying gauge structure of the model the associated fermion content is also different. We make here the first attempt at finding empirical discriminants which would tell these models apart. Demonstrating the power of simple kinematic observables involving samesign leptons, we construct simple yet powerful statistical discriminants.
\end{abstract}

DOI: 10.1103/PhysRevD.100.015022

\section{INTRODUCTION}

Non-Abelian gauge extensions to the Standard Model (SM) are characterized by the presence of heavy charged gauge bosons. They could be a result of an additional $S U(2)$ triplet of gauge bosons or a gauged custodial symmetry like $S U(2) \times S U(2)_{R} \times U(1)_{B-L}$. Such extensions are strongly motivated in the context of explanations for the anomalies observed in the semileptonic decays of the $B$ meson due to heavy vectors. In the event of their confirmation it would be instructive to find the UV origins of these charged states. Similarly, warped extradimensional models [1] are also characterized by gauge extensions corresponding to their solution to softening the corrections to the oblique parameters.

The corrections to the $T$ parameter can be softened by enlarging the gauge symmetry in the bulk to $S U(3)_{c} \times$ $S U(2)_{L} \times S U(2)_{R} \times U(1)_{y}[2,3] .{ }^{1}$ An appropriate choice of the fermion representations also helps suppress contributions in the nonoblique $Z \rightarrow b \bar{b}$ corrections [7,8].

An alternative to enhanced gauge symmetry is to use a deformed metric near the IR brane $[9,10]$, with the softening of the singularity at the IR boundary implying that the Higgs is a bulk scalar field. The line element in this case is

\footnotetext{
${ }^{1}$ A six-dimensional generalization of the Randall-Sundrum (RS) model brings this mass limit down to about 7-8 TeV [4-6].

Published by the American Physical Society under the terms of the Creative Commons Attribution 4.0 International license. Further distribution of this work must maintain attribution to the author(s) and the published article's title, journal citation, and DOI. Funded by SCOAP ${ }^{3}$.
}

$$
\begin{aligned}
d s^{2} & =e^{-2 A(y)} \eta_{\mu \nu} d x^{\mu} d x^{\nu}-d y^{2}, \\
\text { where } A(y) & =k y-\nu^{-2} \log \left(1-y / y_{s}\right)
\end{aligned}
$$

(the limit $\nu \rightarrow \infty$ reverting to the RS geometry). The UV brane is located at $y=0$. The IR brane is, however, located at $y=y_{1}$ with the position of the singularity $\left(y=y_{s}\right)$ located behind it at $y_{s} \simeq y_{1}+\mathcal{O}\left(k^{-1}\right)$. To address the hierarchy one requires $A\left(y_{1}\right) \sim 35$, which fixes the value of $y_{1}$.

There have been several analyses which have been proposed for the discovery of the electroweak charged gauge bosons [11-15]. Typically, irrespective of the underlying model, the most dominant search channel either corresponds to a dijet or a $t b$ final state [16]. In the event of its discovery, it is instructive to develop techniques to probe their underlying gauge origins. These models could also be associated with vectorlike quarks with either standard or exotic electromagnetic charges. We illustrate this in the context of warped extra-dimensional models. In the custodial models, the SM fermion doublets are extended to fields transforming as $(2,2)$. The quark multiplet thus contains exotic $\chi_{5 / 3}$ fermions absent in the noncustodial model. We look at the production of these states, in association with a top, from the decay of the first Kaluza Klein (KK) mode of the $W^{\prime \pm}$ bosons. Cascade decays of the $\chi_{5 / 3}$ fermion lead to two leptons with the same sign. Such final states are also possible in noncustodial scenarios, where a heavy gauge boson decays into a vectorlike quark and a top. However, the two cases are characterized by different kinematics and we demonstrate that simple kinematic variables like the azimuthal separation $\Delta \phi$ between the same-sign leptons and $p_{T}$ combinations of the same-sign leptons are effective in not only suppressing the background but also in distinguishing between the two scenarios. 
The third-generation quarks are likely to have a larger coupling to a $W^{\prime+}$. This can be motivated by solutions to the $B$ anomalies in both standard and extra-dimensional extensions to the SM. Similar coupling could be expected for a $W^{\prime}$ into that to a vector like quark (VLQ)-SM quark pair. Thus, owing to the larger phase space available, the KK $W^{\prime+}$ would decay primarily into the former channel, rendering this the discovery mode. Several dedicated analyses in this direction have used combinations of different variables (both kinematic and substructure) to extract the maximum signal efficiency [11,13-15]. Additionally, ATLAS [16] has an exhaustive analysis for this channel involving jets with different radius parameters to identify the top and bottom candidates. separately. Furthermore, for each $m_{t b}$ bin, the data are classified into signal regions depending on top and $b$-tagging criteria. Depending on the mass of the $W^{\prime}$, only certain signal regions have an enhanced signal selection efficiency. Thus the search can be optimized accordingly leading to a greater signal significance and background rejection. Instead, we perform a very minimal LHC analysis to set up the discovery mode and demonstrate that even simple set of cuts is sufficient achieve an $\sim 3 \sigma$ sensitivity for the process $p p \rightarrow W^{\prime+} \rightarrow t \bar{b}$. The matrix element for this process is generated using MADGRAPH 5.2 [17] using the model files generated by FEYNRULES. To maximize discovery potential, hadronic decay of the top is considered. Generated events are passed on to PYTHIA 8 [18] for showering and hadronization. For $m_{W^{\prime}} \sim 2.5 \mathrm{TeV}$, the decay partons are likely to be associated with very high transverse momentum $\left(p_{T}\right)$ jets. The jet reconstruction radius must be such that the decay products of the top are captured within a cone of radius $R$, with the opening angle roughly being $\sim 2 m_{t} / p_{T}^{t}$, where $p_{T}^{t}$ is the top transverse momentum. It is clear that a radius $R=0.5$ is sufficient for the kinematics under consideration. We use FASTJET [19] with the Cambridge-Aachen [20] jet clustering algorithm and require that the jets satisfy $p_{T}>100 \mathrm{GeV}$. The top candidate is identified among the two leading jets, using the HEP TOP TAGGER [21] algorithm.

Post top identification, we demand that the invariant mass of the two jet system satisfies $2000<m_{j_{0} j_{1}}<3000 \mathrm{GeV}$. To estimate the background, we simulate hard QCD processes in the following kinematic regime: Require the scalar sum of the visible transverse momenta to be $\sum p_{T}^{j}>500 \mathrm{GeV}$ and the invariant mass of the outgoing partons to be $\hat{m}_{\text {jets }}>800 \mathrm{GeV}$. Taking into account the width of the resonance as well as the mass resolution of the final states, the background is generated with these choices of parameters. These values are chosen as the decay constituents of $W^{\prime}$ are likely to have a $p_{T}$ of at least 500 $600 \mathrm{GeV}$ each. This reduces the QCD cross section to $10^{5} \mathrm{fb}$. Furthermore, after the selection criteria detailed above are imposed, the QCD background has a fake rate of $0.1 \%$ while the signal has an acceptance of $13 \%$. The production cross section for the $W^{\prime}$ is chosen to be $20 \mathrm{fb}^{2}$ This leads to a rough sensitivity of $S / \sqrt{B} \sim 3$ with $\sim 400 \mathrm{fb}^{-1}$ of data. It is to be stressed that this acceptance can be significantly improved with even less luminosity. However, it has been considered extensively and is not the goal of this paper. The objective behind this section was to demonstrate that even the most basic cuts is sufficient to obtain a reasonable signal acceptance. Post this discovery, it is then necessary to extract the origin of the heavy charged object: whether the origin of the heavy gauge state is due to a SM gauge symmetry or due to and extended gauge group. One simple way is to look at the charge of the heavy fermion the $W^{\prime}$ decays into, as determined by analyzing the multileptonic final state.

For custodial models, the electroweak gauge group is extended to $S U(2)_{L} \times S U(2)_{R} \times U(1)_{B-L}$. The $(t, b)$ doublet is replaced by a bidoublet represented by

$$
Q_{3} \equiv\left[\begin{array}{ll}
\chi_{5 / 3} & t \\
\chi_{2 / 3}^{\prime} & b
\end{array}\right]
$$

where $\chi$ and $\chi^{\prime}$ are exotic fermions with electromagnetic charges $5 / 3$ and $2 / 3$, respectively. The crucial difference between the noncustodial and the custodial models is the presence of the charge $5 / 3$ state. For simplicity we consider $t_{R}$ to be a gauge singlet and consider the decay of the $W^{\prime}$ is into $\chi_{5 / 3} \bar{t}$. The $\chi_{5 / 3}$ can further decay into $W t$ resulting in a $W t \bar{t}$ final state. Considering a total leptonic final state, this leads to three leptons with two leptons of the same sign.

For noncustodial models, the gauge structure is SM-like and hence the heavy fermions also have charges $Q=2 / 3$ and $-1 / 3$. The aforementioned three lepton final state can arise in two ways: (a) The VLQ decays into a $t Z$ or $b Z$ as the case may be. Thus, the net final state from the gauge KK state is $t \bar{b} Z$. Assuming both the top and the $Z$ decay leptonically, we have a three-lepton final state with two leptons of the same sign. This case can however be distinguished using a $Z$ mass veto for two leptons with opposite sign. (b) If the KK fermion decays into $W \bar{b}$ $(Q=2 / 3)$ and $W^{-} t(Q=-1 / 3)$, the overall final state from the gauge KK would be $W \bar{b} b$ and $W \bar{t} t$, respectively. The former leads to only a single isolated hard lepton, while the latter may lead to three leptons and two $b$-tagged jets.

Figure 1 shows the Feynman diagrams for both scenarios leading to a $t t W$ final state. For the purpose of discrimination between the two cases we consider a multilepton final state corresponding to the leptonic decays of $W$ and the $t$.

\section{EVENT SELECTION}

The signal event is characterized by the presence of three leptons (electron or muons) and at least a single jet.

\footnotetext{
${ }^{2}$ This is the typical value for the production cross section for charged bosons in extra-dimensional models.
} 

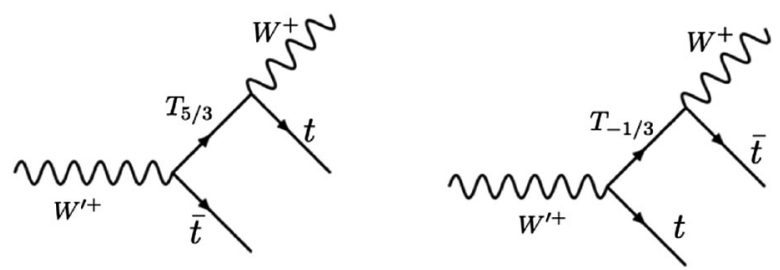

FIG. 1. Feynman diagrams for models with exotic VLQ with charge 5/3 (left) and charge $-1 / 3$ (right). For both cases the VLQ are denoted as $T$.

The isolated leptons for each event are required to satisfy $\sum_{i} p_{T}^{l_{i}}>600 \mathrm{GeV}$ and $\eta^{L_{1,2,3}} \leq 2.5$. Note that while the presence of the third lepton is not crucial to the discrimination, it aids in discriminating against the $t \bar{t}$ background. To identify the isolated leptons, we adapt the following isolation criterion: In order to ensure that they are from the signal and free from nearby hadronic activity, we demand that the scalar sum of transverse momenta of jets within a cone of size $\Delta R(l, j) \leq 0.3$ around the lepton $l$ is less than $10 \%$ of the $p_{T}^{l}$ is the corresponding lepton. This simplified isolation criterion rests on the assumption that the acceptance efficiencies for the electrons and muons are the same. We adapt a simple isolation criterion which assumes equal efficiencies for the electron and muon. The jets, on the other hand, are clustered with the anti-kt algorithm [22] using FASTJET [19]. We consider the following four different combinations of benchmark points:

$$
\begin{array}{ll}
\text { BP1 }::(3000,1500), & \text { BP2 }::(3000,2000), \\
\text { BP3 }::(3500,1500), & \text { BP4 }::(3500,2000),
\end{array}
$$

where masses are expressed as $\left(m_{W^{\prime}}, m_{V L Q}\right)$. Table I gives the total signal and background efficiencies. The main background is $t \bar{t}+$ jets, where the third same-sign leptons are either due to instrumental effects or due to the decay of the $b$ quark [23]. In order to populate the signal phase space the events are simulated requiring the minimum invariant $p_{T}$ of the outgoing partons to be $1000 \mathrm{GeV}$. This along with the leptonic decay of the tops reduces the effective cross section to $\sim 9 \mathrm{fb}$. On the other hand, the $t t w$ background has a much smaller cross section $\sim 0.05 \mathrm{fb}$. The effective cross section of $t t W$ is smaller than that of the signal, making $t \bar{t}$ the dominant background.

TABLE I. Table giving efficiencies for the background and the different signal benchmarks. For the BPs the upper cell corresponds to the model with the presence of $Q=5 / 3 \mathrm{VLQ}$, while the bottom one corresponds to $Q=-1 / 3 \mathrm{VLQ}$.

\begin{tabular}{lllll}
\hline \hline Background & BP1 & BP2 & BP3 & BP4 \\
\hline$t t W: 0.03$ & 0.39 & 0.43 & 0.38 & 0.44 \\
$t \bar{t}: 0.049$ & 0.26 & 0.32 & 0.27 & 0.33 \\
\hline \hline
\end{tabular}
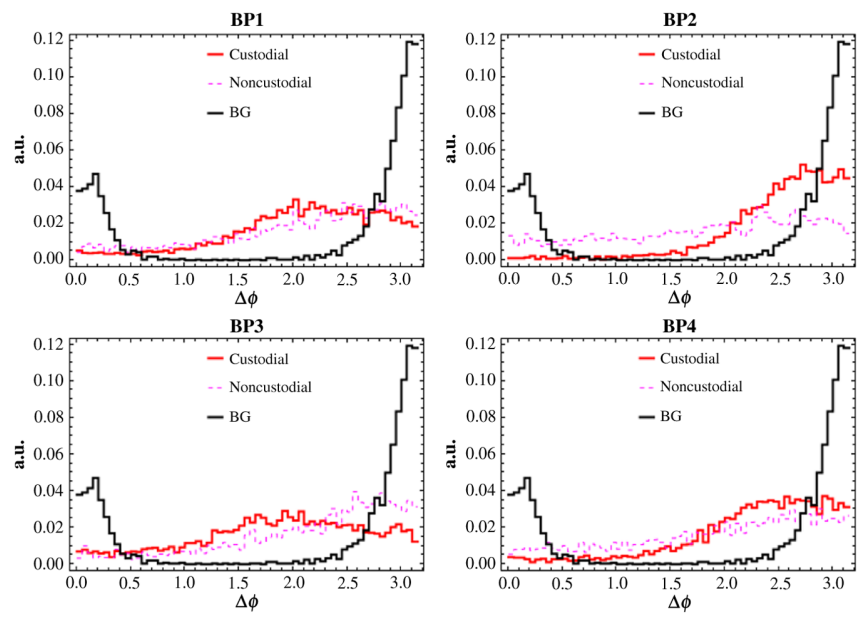

FIG. 2. $\Delta \phi$ between the same-sign leptons for the four benchmark points.

\section{VARIABLES FOR DISCRIMINATION}

In order to distinguish the two scenarios, it is necessary to understand the kinematic features for the two gauge structures. Since the signal is characterized by the presence of two leptons of the same sign, it is useful to construct variables using these two leptons.

\section{A. $\Delta \phi_{l^{ \pm} l^{ \pm}}$between the same-sign leptons}

For the custodial case, the two same-sign leptons originate from the decay of the $5 / 3$ state. As a result the $\Delta \phi$ between them would depend on the boost of the VLQ. For the noncustodial case, on the other hand, one of them is due to the decay of the VLQ while the other is due to the top originating from the heavy charged vector boson. Since the VLQ and the top from the charged boson are produced back to back, the same-sign leptons from them are also broadly separated. As a result the utility of this variable to segregate the two cases depends on the benchmark point used. For the background the same-sign lepton is due to radiation off one of the tops and hence has a very distinct distribution where the $\Delta \phi_{l^{ \pm} l^{ \pm}}$are either back to back or in the same direction. This is extremely useful in distinguishing the background from the signal. Figure 2 gives the distribution of this variable for the different benchmark points and the background. The noncustodial models are characterized by a fairly similar distribution for all the benchmark points. It can be seen that the efficiency of this variable is better for BP2 over others and can be attributed to the larger boost of $1 \mathrm{TeV}$ VLQ states from the decay of $W^{\prime}$.

\section{B. $p_{T}^{\min }$ of the same-sign lepton combination $\left[p_{T}^{\min }=\min \left(p_{T}^{l_{1}^{ \pm}}, p_{T}^{l_{2}^{ \pm}}\right)\right]$}

For the noncustodial models one of the same-sign leptons is due to the SM $W$ originating from the decay 

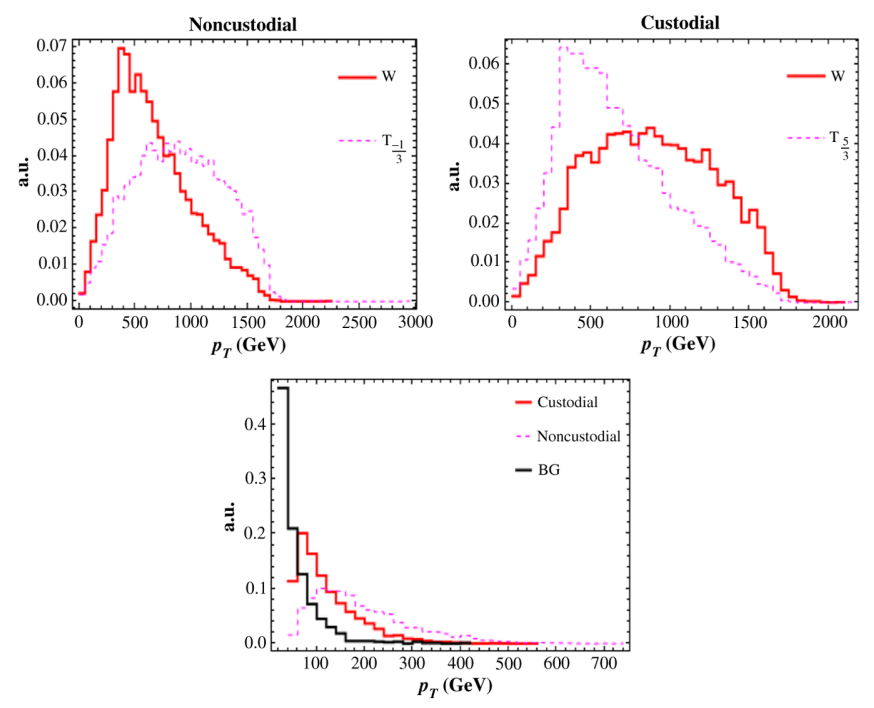

FIG. 3. Plots in the top row give the parton $p_{T}$ of the decay products of the VLQ for noncustodial (left) and for custodial (right) scenarios. The bottom plot gives the distribution of the minimum transverse momentum between the same-sign lepton combination $p_{T}^{\min }=\min \left(p_{T}^{l_{1}^{ \pm}}, p_{T}^{l_{2}^{ \pm}}\right)$.

of the VLQ while the other is due to the top from the heavy gauge boson vertex. Due to the boost of the top, the corresponding lepton from this top is characterized by larger $p_{T}$ than the one due the $W$ from the decay of the VLQ. The left plot of Fig. 3 gives the comparison of parton level $p_{T}$ for the $W$ and the top from the VLQ. While the $W$ is characterized by smaller transverse momentum, it is only shared between lepton and the neutrino. Resultantly, the $p_{T}^{\min }$ of the same-sign lepton combination is likely to have a momentum distribution peaking at relatively larger values as shown by the dashed pink line at the bottom plot of Fig. 3.

For custodial models on the other hand, the two samesign leptons are from the decay products of the VLQ. The right plot of Fig. 3 gives a comparison of the $p_{T}$ for $W$ and the top from $\chi_{5 / 3}$ VLQ. Since the $p_{T}$ is shared between three objects, $b$ jet, lepton and neutrino, the corresponding lepton is characterized by relatively lower $p_{T}$ than in the case when the decay proceeds due a noncustodial scenario. This is evident by the distribution of the solid red line in the bottom plot in Fig. 3 .

Given the two distributions, it is necessary to develop a quantitative measure to distinguish them. We assume one particular hypothesis, say, $H_{T}$, to be true, which is to be tested against the alternative hypothesis, say, $H_{A}$. To estimate the number of events $N$ required to disfavour a given spin hypothesis $H_{A}$ to some factor $R$, we solve

$$
\frac{1}{R}=\frac{p\left(H_{A} \mid N \text { events from } H_{T}\right)}{p\left(H_{T} \mid N \text { events from } H_{T}\right)},
$$

where $R$ is an integer and implies that the alternative hypothesis $H_{A}$ is disfavored at $1: R$ odds in favor of $H_{T}$.
Following Refs. [24,25], we present results for $R=20$ and 1000 . The $N$ events are characterized by their values of either one or a set of observables $\mathcal{O}_{i}$. In the first instance we choose two possibilities: either $\mathcal{O}_{1}=\Delta \phi$ or $\mathcal{O}_{1}=p_{T}^{\min }$ between the same-sign lepton candidates. We find that while the former is useful for background discrimination, the latter is more efficient for distinguishing the two models. Following this we adapt an analysis involving both the variables simultaneously. Following the steps in [25] for the discrete implementation of Kullback-Leibler divergence [24], Eq. (3) becomes

$$
\log \left(\frac{1}{R}\right)=\sum_{i=1}^{K}\left[\mu_{j}^{(T)} \log \frac{\mu_{j}^{(A)}}{\mu_{j}^{(T)}}+\mu_{j}^{(T)}-\mu_{j}^{(A)}\right],
$$

where $\mu_{j}$ is the expectation value for the number of events in the $j$ th bin for a given hypothesis. To translate the above expression into the number of events (and hence the integrate luminosity $\mathcal{L}$ ) required to separate $H_{A}$ from $H_{T}$ at $1: R$, we use

$$
\mu_{j}^{(X)}=\mathcal{L} \sigma_{\mathrm{tot}}^{\left(W^{\prime}\right)} \text { B.R. }\left(W^{\prime} \rightarrow l^{+} l^{-} l^{+}+X\right) \epsilon_{j}^{(X)},
$$

where $X$ denotes jets and missing energy and $\epsilon_{j}$ is the collider acceptance efficiency for the $j$ th bin. ${ }^{3}$

Using Eqs. (4) and (5) and $N_{R}=\mathcal{L} \sigma_{\mathrm{tot}}$ B.R. $\left(W^{\prime} \rightarrow\right.$ $\left.l^{+} l^{-} l^{+}+X\right)$, the number of events $N_{R}$ of the true hypothesis $H_{T}$ to disfavor $H_{A}$ at $1: R$ odds is

$$
N_{R}=\frac{\log R}{\sum_{j=1}^{K}\left[\epsilon_{j}^{(T)} \log \frac{\epsilon_{j}^{(T)}}{\epsilon_{j}^{(A)}}+\epsilon_{j}^{(A)}-\epsilon_{j}^{(T)}\right]} .
$$

Note that we assume $\sigma_{\text {tot }}$ B.R. $\left(W^{\prime} \rightarrow l^{+} l^{-} l^{+}+X\right)$ the same for both the hypotheses which is a reasonable approximation.

\section{RESULTS OF THE ANALYSIS}

We employ this analysis for the different benchmark masses in Eq. (2). Upper limits exist on $\sigma\left(p p \rightarrow W^{\prime}\right) \times$ B.R. $\left(W^{\prime} \rightarrow t b\right)$ from direct searches on the $t b$ final state [16] where there is a rough upper bound on $\sigma\left(p p \rightarrow W^{\prime}\right) \times$ B.R. $\left(W^{\prime} \rightarrow t b\right)<90 \mathrm{fb}$ for masses between 3 and $3.5 \mathrm{TeV}$. We assume a production cross section of $60 \mathrm{fb}$ for simplicity. Table II gives the results of the statistical discussion using both $p_{T}^{\min }-\Delta \phi_{l^{ \pm} l^{ \pm}}$. We present results for both $R=20$ (black) and $R=1000$ (red). The probabilities are computed by constructing bins of sizes $(0.7,145)$ in the $\Delta \phi_{l^{ \pm} l^{ \pm}}-p_{T}^{\min }$ over the range $[0-\pi, 0-570]$. While $p_{T}^{\min }$ of the same-sign lepton combination is extremely

\footnotetext{
${ }^{3} \mathrm{We}$ assume that $\sigma_{\mathrm{tot}}^{\left(W^{\prime}\right)}$ is the same for both the hypotheses $X=A, T$.
} 
TABLE II. Table gives the expected number of events $N_{R}=\mathcal{L} \sigma_{\text {tot }}^{(X)}$, to disfavor the column hypothesis $\left(H_{A}\right)$ in favor of the row hypothesis $\left(H_{T}\right)$ by a factor of $R=20$ (black) and $R=1000$ (red) at the $13 \mathrm{TeV}$ LHC. Both $p_{T}^{\min }$ and $\Delta \phi_{l^{ \pm} l^{ \pm}}$ variables are used in this case.

\begin{tabular}{|c|c|c|c|c|c|}
\hline \multicolumn{3}{|c|}{ BP1 } & \multicolumn{3}{|c|}{ BP2 } \\
\hline$N_{R}$ & Cust. & Noncust. & $N_{R}$ & Cust. & Noncust. \\
\hline Cust. & $\infty$ & $\begin{array}{c}9 \\
21\end{array}$ & Cust. & $\infty$ & $\begin{array}{l}10 \\
22\end{array}$ \\
\hline Noncust. & $\begin{array}{l}12 \\
25\end{array}$ & $\infty$ & Noncust. & $\begin{array}{c}7 \\
17\end{array}$ & $\infty$ \\
\hline & BP3 & & & BP4 & \\
\hline$N_{R}$ & Cust. & Noncust. & $N_{R}$ & Cust. & Noncust. \\
\hline Cust. & $\infty$ & $\begin{array}{c}9 \\
20\end{array}$ & Cust. & $\infty$ & $\begin{array}{l}11 \\
27\end{array}$ \\
\hline Noncust. & $\begin{array}{l}11 \\
23\end{array}$ & $\infty$ & Noncust. & $\begin{array}{l}10 \\
23\end{array}$ & $\infty$ \\
\hline
\end{tabular}

useful in distinguishing the two scenarios, it is not as effective as $\Delta \phi_{l^{ \pm} l^{ \pm}}$for background discrimination. Note that the conclusions using both variables are expected to be similar within statistical fluctuations to those which take only $p_{T}^{\min }$ into account. This is because $p_{T}^{\min }$ plays the dominant role for the discrimination in both cases while $\Delta \phi_{l^{ \pm} l^{ \pm}}$is practically a dummy variable as far as the discrimination between the two signal possibilities is concerned. We reiterate that the role $\Delta \phi_{l^{ \pm} l^{ \pm}}$is primarily restricted to segregating both the signal possibilities from the background. Given the drastically different distributions of $\Delta \phi_{l^{ \pm} l^{ \pm}}$in Fig. 2 for the signal and background, distinguishing them is fairly straightforward. Note that the distribution in black is plotted for the dominant $t \bar{t}$ background, though $t t W$ also exhibits a similar pattern.

Finally we also demonstrate the spin discrimination analysis when the spectrum is fairly compressed. We add another benchmark point BP0 :: $(3500,3100)$ and note that, similar to the results in Table II, 5-6 events are sufficient to discriminate custodial models from the noncustodial ones at 1:20 odds.

\section{DISCUSSIONS}

The results in Table II can be converted to the required luminosity by simply assuming a production cross section for the $W^{\prime}$ and the branching fraction for the VLQ into the corresponding states. For simplicity we discuss the results for $R=20$ and assume equal production cross sections and branching fractions for both the models. If we assume a production cross section of $60 \mathrm{fb}$ and the following branching fractions: B.R. $\left(W^{\prime} \rightarrow T t\right) \sim 50 \%$ and B.R. $(T \rightarrow t W) \sim 33 \%$, where $T$ is a VLQ with charge either $5 / 3$ or $1 / 3$. Consider BP1 where nine events are necessary to reject the noncustodial hypothesis. This benchmark for custodial models has an acceptance efficiency of $39 \%$ as shown in Table I. Taking into account the leptonic branching fraction of the three $W$ bosons viz. $\left(0.2^{3}\right)$, then nine events can be accumulated with $300 \mathrm{fb}^{-1}$ of data. Similarly the analysis can be applied to any benchmark point and any scenario with a similar particle content but different values for the production and branching fractions. Smaller branching fractions which are just out of reach of the HL-LHC could pave the way for their exploration at future colliders.

Heavy charged gauge bosons are a characteristic feature of several extensions beyond the Standard Model. Corresponding to the gauge origins of these heavy vectors, the representation of the fermion content also differ. Using this as a motivation, we present a methodology to distinguish the two cases in the event of a discovery. We demonstrate the effectiveness of this technique by using a statistical tool which utilities simple kinematic variables like $\Delta \phi$ between the same-sign leptons and $p_{T}$. Given the generic nature of the method, the analysis and the corresponding results can also be extended with similar gauge and fermion content.

\section{ACKNOWLEDGMENTS}

A. M. I. was supported in part by MIUR under Project No. 2015P5SBHT and by the INFN research initiative ENP. The work of K. S. is partly supported by a project grant from the Indo-French Centre for the Promotion of Advanced Research (Project No. 5904-2).
[1] L. Randall and R. Sundrum, Phys. Rev. Lett. 83, 3370 (1999).

[2] K. Agashe, A. Delgado, M. J. May, and R. Sundrum, J. High Energy Phys. 08 (2003) 050.

[3] K. Agashe, R. Contino, L. Da Rold, and A. Pomarol, Phys. Lett. B 641, 62 (2006).
[4] M. T. Arun and D. Choudhury, J. High Energy Phys. 09 (2015) 202.

[5] M. T. Arun and D. Choudhury, J. High Energy Phys. 04 (2016) 133.

[6] M. T. Arun and D. Choudhury, Nucl. Phys. B923, 258 (2017). 
[7] H. Davoudiasl, S. Gopalakrishna, E. Ponton, and J. Santiago, New J. Phys. 12, 075011 (2010).

[8] A. M. Iyer, K. Sridhar, and S. K. Vempati, Phys. Rev. D 93, 075008 (2016).

[9] J. A. Cabrer, G. von Gersdorff, and M. Quiros, Phys. Lett. B 697, 208 (2011).

[10] J. A. Cabrer, G. von Gersdorff, and M. Quiros, J. High Energy Phys. 05 (2011) 083.

[11] K. Agashe, S. Gopalakrishna, T. Han, G.-Y. Huang, and A. Soni, Phys. Rev. D 80, 075007 (2009).

[12] K. Agashe, H. Davoudiasl, S. Gopalakrishna, T. Han, G.-Y. Huang, G. Perez, Z.-G. Si, and A. Soni, Phys. Rev. D 76, 115015 (2007).

[13] J. de Blas, A. Delgado, B. Ostdiek, and A. de la Puente, Phys. Rev. D 86, 015028 (2012).

[14] M. Frank, A. Hayreter, and I. Turan, Phys. Rev. D 83, 035001 (2011).

[15] A. Carmona, E. Ponton, and J. Santiago, J. High Energy Phys. 10 (2011) 137.

[16] M. Aaboud et al. (ATLAS Collaboration), Phys. Lett. B 781, 327 (2018).
[17] J. Alwall, R. Frederix, S. Frixione, V. Hirschi, F. Maltoni, O. Mattelaer, H. S. Shao, T. Stelzer, P. Torrielli, and M. Zaro, J. High Energy Phys. 07 (2014) 079.

[18] T. Sjostrand, S. Ask, J. R. Christiansen, R. Corke, N. Desai, P. Ilten, S. Mrenna, S. Prestel, C. O. Rasmussen, and P.Z. Skands, Comput. Phys. Commun. 191, 159 (2015).

[19] M. Cacciari, G. P. Salam, and G. Soyez, Eur. Phys. J. C 72, 1896 (2012).

[20] Y. L. Dokshitzer, G. D. Leder, S. Moretti, and B. R. Webber, J. High Energy Phys. 08 (1997) 001.

[21] T. Plehn, M. Spannowsky, M. Takeuchi, and D. Zerwas, J. High Energy Phys. 10 (2010) 078.

[22] M. Cacciari, G. P. Salam, and G. Soyez, J. High Energy Phys. 04 (2008) 063.

[23] S. Chatrchyan et al. (CMS Collaboration), Phys. Rev. Lett. 112, 171801 (2014).

[24] C. Athanasiou, C. G. Lester, J. M. Smillie, and B. R. Webber, J. High Energy Phys. 08 (2006) 055.

[25] B. C. Allanach, D. Bhatia, and A. M. Iyer, Eur. Phys. J. C 77, 595 (2017). 